\title{
Distribution of the atypical pathogens of community-acquired pneumonia to disease severity
}

\author{
Cheng Gong", Tiegang Zhang", Ming Luo, Aihua Li, Mei Dong, Maozhong Li, Yiting Wang, Fang Huang \\ Institute for Immunization and Prevention, Beijing Center for Disease Prevention and Control, Beijing Research Center for Preventive Medicine, \\ Beijing 100013, China \\ Contributions: (I) Conception and design: F Huang; (II) Administrative support: None; (III) Provision of study materials: None; (IV) Collection \\ and assembly of data: M Luo, A Li, M Dong, M Li, Y Wang; (V) Data analysis and interpretation: C Gong, T Zhang; (VI) Manuscript writing: All \\ authors; (VII) Final approval of the manuscript: All authors. \\ \#These authors contributed equally to this work. \\ Correspondence to: Fang Huang, MD, Institute for Immunization and Prevention, Beijing Center for Disease Prevention and Control, Beijing \\ Preventive Medicine Research Center, 16 Hepingli Middle Avenue, Dongcheng district, Beijing 100013, China. Email: huangfang7032@126.com.
}

Background: To investigate the epidemiological characteristics of 11 atypical pathogens of communityacquired pneumonia (CAP) among Chinese, and to determine whether or not there is an association between these pathogens and the severity of illness.

Methods: We conducted a surveillance study for CAP in 30 hospitals of Beijing. Epidemiological data and clinical specimens were systematically collected from enrolled CAP patients. The detection for 11 atypical pathogens [9 respiratory viruses, Mycoplasma pneumoniae (MP) and Cblamydophila pneumoniae (CP)] was performed. Risk factors of severe CAP and death in Hospital were evaluated.

Results: A total of 6,008 CAP patients [including 1,071 severe CAP (SCAP)] were enrolled. The overall detection rate of the 11 atypical pathogens was $42.4 \%$ among 1,925 child CAP $(39.9 \%$ among 274 child SCAP), and $25.8 \%$ among 4,083 adult CAP (22.8\% among 797 adult SCAP). The most frequent atypical pathogen among child SCAP was parainfluenza virus (10.2\%) followed by respiratory syncytial virus (RSV) (8.4\%). However, the most frequent atypical pathogen among adult SCAP was influenza virus $(8.9 \%)$ followed by parainfluenza virus (3.8\%). Multivariate analyses showed that the important predictors for SCAP were an age $\leq 9$ years, an age $\geq 65$ years and co-existing diseases. These factors, except an age $\leq 9$ years, were also predictors of death in Hospital. None of these 11 atypical pathogens was included as the risk factors of SCAP or death in Hospital.

Conclusions: Although these 11 atypical pathogens were the common causes of CAP (including SCAP) among Chinese, they were not observed to increase risks for SCAP or death in Hospital.

Keywords: Community-acquired pneumonia (CAP); severe community acquired pneumonia; respiratory pathogen; atypical respiratory pathogen; surveillance

Submitted Feb 07, 2018. Accepted for publication Sep 25, 2018.

doi: $10.21037 /$ jtd.2018.10.50

View this article at: http://dx.doi.org/10.21037/jtd.2018.10.50

\section{Introduction}

Community-acquired pneumonia (CAP) is a major cause of hospitalization and death worldwide. Its annual incidence ranges from 2.7-10 per 1,000 persons in European countries (1), 2.67-12 per 1,000 persons in the US $(2,3)$ and $3.4-42.9$ per 1,000 persons in Japan $(2,3)$.
CAP can be caused by bacteria, viruses, fungi and parasites etc. (4). According to Jain's reports $(5,6)$, virus infections in CAP patients, especially in adult cap, were more commonly than we expected. In China, there are two multiple center, population based studies that report the characteristics of bacteria, Mycoplasma pneumoniae (MP) 
and Chlamydophila pneumoniae (CP) among Chinese adults with CAP $(7,8)$. However, other existing studies on the viral characteristics of CAP are limited to single sites $(8,9)$.

In Beijing, a Respiratory Pathogens Surveillance System (RPSS) has been established since 2014 to monitor the contagion of respiratory pathogens associated with CAP by the Beijing Center for Disease Prevention and Control (BJCDC). In this multiple center, population based, prospective surveillance study, systematic enrollment and comprehensive microbiological investigation were conducted to explore the epidemiological characteristics of nine frequent respiratory viruses, $\mathrm{MP}$ and $\mathrm{CP}$ among $\mathrm{CAP}$ patients in China, to find the risk factors for SCAP and death in hospital, and to determine whether or not there is an association between these pathogens and the severity of illness.

\section{Methods}

\section{Study population}

In the RPSS study, a total of 30 hospitals from all 16 districts of Beijing were recruited as sentinel hospitals (Table S1), which includes 12 general hospitals and 18 district level hospitals. Under the protocol of the study, approximately 10 CAP patients were required to be enrolled from each hospital every month. These 10 selected patients of each month were required to be distributed as evenly throughout the month as possible. Patients were included if they presented or were admitted to one of the 30 study hospitals and further met the following two conditions: they resided in 1 of the 16 districts in the study catchment areas, and had evidence of CAP according to Guidelines of Diagnosis and Treatment for Community Acquired Pneumonia among Adults in China (released in 2013 by Chinese Thoracic Society, Chinese Medicine Association) (10). CAP was defined as a new or aggravated infiltrate in chest radiographs obtained within 72 hours before or after presentation, which was accompanied by one of the following clinical characteristics: new cough or sputum production, aggravated underlying respiratory disease, fever $\left(>37.8^{\circ} \mathrm{C}\right)$, hypothermia $\left(<35.6^{\circ} \mathrm{C}\right)$, leukocytosis $\left(>10 \times 10^{9} / \mathrm{L}\right)$, leukopenia $\left(<4 \times 10^{9} / \mathrm{L}\right)$ or pulmonary consolidation or moist rale. The immunocompetent and immunocompromised patients with CAP were all included, immunocompromised circumstances referred to: congenital immunodeficiency syndrome, receiving chemotherapy, or anti-rejection therapy, or corticosteroid therapy, human immunodeficiency virus (HIV) infection with a CD4 cell count of $>200$ per cubic millimeter.
Patients were excluded if they had been hospitalized recently ( $<28$ days for immunocompetent patients and $<90$ days for immunocompromised patients), had already been enrolled in the RPSS study within the previous 28 days, or had a clear alternative diagnosis of a respiratory disorder. We also excluded patients if they had lung cancer or tuberculosis, pneumoconiosis, noninfectious interstitial lung disease, pulmonary edema, pulmonary atelectasis, pulmonary thromboembolism, pulmonary infiltration with eosinophilia, or pulmonary vasculitis.

According to the Guideline on the Management of Community Acquired Pneumonia among Children in China (released in 2013 by Chinese pediatric society, Chinese Medicine Association) $(11,12)$. The children with CAP were identified as the SCAP cases if they had at least one of the following clinical features: severely disturbed general conditions, refusal to eat, dehydration, consciousness impairment, the respiratory rate increased to exceed the threshold level ( $>50$ per minute for children over 1 year of age ( $>70$ for infant less than 1 year of age), cyanosis, dyspnea, more than 2 lung lobes or more than two thirds of lung infiltrated, pleural effusion, $\mathrm{SaO}_{2}>0.92$, nonpulmonary complications.

According to The Guideline of Diagnosis and Treatment for Community Acquired Pneumonia among Adults in China (released in 2013 by Chinese Thoracic Society, Chinese Medicine Association) (10). Adults with CAP were identified as SCAP cases if they had at least one of the following: consciousness impairment, an increased respiratory rate exceeding the threshold level $(>30$ per minute), $\mathrm{PaO}_{2}<60 \mathrm{mmHg}, \mathrm{PaO}_{2} / \mathrm{FiO}_{2}<300$, mechanical ventilation being needed, artery systolic pressure of less than $90 \mathrm{mmHg}$, septic shock, infiltration of more than one lung lobe or an infiltrated area increased by $50 \%$ during 48 hours after admission, oliguresis (urine output $<20$ milliliter per hour) or acute renal failure needing dialysis. Compared to Infectious Diseases Society of America/American Thoracic Society Consensus Guidelines on Management of Community-acquired Pneumonia in Adults Version 2007 (13), the Chinese criteria of SCAP didn't include three minor criteria: leukopenia (WBC count, $<4,000 \mathrm{~mm}^{3}$ ), thrombocytopenia (platelet count, $<1,000,000 \mathrm{~mm}^{3}$ ), hypothermia (core temperature, $<36^{\circ} \mathrm{C}$ ).

\section{Specimen and data collection}

Oropharyngeal swabs were obtained from the enrolled patients as soon as possible after presentation with 


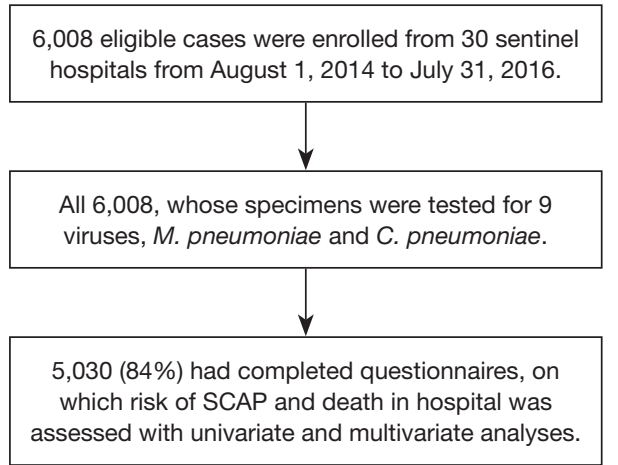

Figure 1 Flow chart of enrollment of CAP patients with radiographic evidence. CAP, community-acquired pneumonia; SCAP, severe CAP.

symptoms. In cases of patients with sputum production, sputum specimens were also obtained. Endotracheal aspirates, and bronchoalveolar-lavage specimens that were obtained for clinical care were also analyzed for the study. Only specimens obtained within 72 hours before or after admission were included. The enrolled patients, their care providers, or both were interviewed using a standardized questionnaire. The following data were collected systematically: demographic information, epidemiology information, underlying diseases, clinical symptoms and signs, laboratory findings, radiology findings, first and final diagnosis, clinical treatment and intervention measures, complications, prognosis.

\section{Laboratory examinations}

Clinical specimens were collected from the enrolled cases at each sentinel hospital and sent to BJCDC for laboratory diagnostic testing. Total nucleic acids (RNA and DNA) were extracted from the respiratory specimens (Thermo Scientific ${ }^{\mathrm{TM}}$ KingFisher ${ }^{\mathrm{TM}}$ Flex Magnetic Particle Processors, Thermo Fisher). The testing for a panel of atypical pathogens, including respiratory viruses, MP and CP, were performed on the extracted nucleic acids. The respiratory viruses included influenza virus A and B (FLU A, FLU B), influenza virus AH1N1 2009 pandemic and AH3N2 (AH1N1 2009, AH3N2), respiratory syncytial virus (RSV), parainfluenza virus 1, 2, 3, 4 (PIV $1,2,3,4)$, adenovirus (AdV), human rhinovirus (HRV), human metapneumovirus (HMPV), human coronavirus 229E/NL63, OC43/HKU1 (CoV 229E/NL63, OC43/ HKU1), human bocavirus ( $\mathrm{HBoV})$, human enterovirus
(EV). All atypical pathogens were tested with the use of the commercial real-time PCR based kits (Multiplex Combined Real-time PCR Detection Kit for Respiratory Viruses, Multiplex Combined Real-time PCR Detection Kit for Respiratory Bacteria, Jiangsu Uninovo Biological Technology Co. Ltd., China).

The aforementioned viral pathogens, MP and CP were determined to be present if they were detected in at least one of the specimens.

\section{Statistical analysis}

Continuous variables were summarized as medians with interquartile ranges. For categorical variables, the percentages of patients in each category were calculated. For analysis convenience, the pathogens were grouped as follows: $\mathrm{CoV}$ 229E/NL63, CoV OC43/HKU1 (CoV), PIV1, 2, 3 and 4(PIV), FLUA, AH1N1 2009, AH3N2, and Flu B (FLU). We used a chi-square test or Fisher's exact test, as appropriate, to compare the percentages of pathogens detected and demographic characteristics between sub groups of NSCAP and SCAP, and between sub groups of death cases (only including death in hospital) and those who recovered. We used multiple binary logisticregression analysis to identify independent predictors of SCAP and death in hospital. The outcomes were predicted with the use of factors such as age, sex, smoke, underlying diseases (chronic lung disease, cardiovascular disease, diabetes mellitus, chronic liver diseases, chronic kidney disease, immunosuppression, neurologic disorder, and other system disease), and pathogens detected (Flu, RSV, HRV, AdV, HBoV, EV, HMPV, PIV, CoV, MP and CP). Age group was transferred to a dummy variable with 18-44 years old as reference category. Forward Stepwise method (likelihood ratio) was used for model building. Variables were included into the model as their $\mathrm{P}$ values less than 0.05 and removed as their $\mathrm{P}$ values more than 0.1 . A $\mathrm{P}$ value of less than 0.05 (two tail) was considered to indicate statistical significance. All analyses were performed with the use of SPSS software for Windows (release 17.0).

\section{Results}

\section{Study population}

During the study period, a total of 6,008 CAP patients confirmed by radiological diagnoses were enrolled into this study from 30 hospitals in Beijing (Figure 1). The median 
age of the patients was 52 years old (interquartile range, 7 to 75$)$. Among the 6,008 CAP cases, 2,442 patients (40.3\%) were female, 1,925 patients $(32.1 \%)$ were children (with an age of $<18$ years old), 2,410 patients (40.1\%) had underlying conditions (with chronic lung disease and cardiovascular disease as the most common), 695 patients (11.6\%) required intensive care, and 168 (2.8\%) died (Table 1).

\section{Detection of pathogens}

Of all 6,008 CAP patients enrolled, an oropharyngeal swab was obtained from 3,770 patients $(62.7 \%)$, a sputum sample from 1,879 [31.3\%, eligible sputum specimens from 823 (43.8\%)], a bronchoalveolar-lavage specimen from 308 $(5.1 \%)$, and an endotracheal aspirate from $51(0.8 \%)$.

A pathogen was detected in 819 of all 1,925 CAP children (42.5\%), as follows: $\geq 1$ viruses in $648(33.7 \%)$, $\mathrm{MP} / \mathrm{CP}$ in $126(6.5 \%)$ and virus-MP/CP co-detections in 45 (2.3\%). However, among the 274 SCAP children, A pathogen was detected in $109(39.8 \%)$, as follows: $\geq 1$ viruses in $90(32.8 \%), \mathrm{MP} / \mathrm{CP}$ in 12 (4.4\%) and virus-MP/CP codetections in 7 (2.6\%) (Figure 2).

A pathogen was detected in 1,053 of all 4,083 CAP adults (25.8\%), as follows: $\geq 1$ viruses in 835 (20.5\%), MP/ $\mathrm{CP}$ in 193 (4.7\%) and virus-MP/CP co-detections in 25 (0.6\%). However, among the 797 SCAP adults, A pathogen was detected in $182(22.8 \%)$, as follows: $\geq 1$ viruses in $175(22.0 \%), \mathrm{MP} / \mathrm{CP}$ in $4(0.5 \%)$ and virus-MP/CP codetections in $3(0.4 \%)$ (Figure 2$)$. the overall detection rates of 11 atypical pathogens among adult CAP and among adult SCAP, all decreased significantly than those of child CAP and child SCAP (42.4\% vs. $25.8 \%, \mathrm{P}<0.001 ; 42.4 \%$ vs. $25.8 \%, 39.9 \%$ vs. $22.9 \%, \mathrm{P}<0.001)$.

Of the 1,925 CAP children, the most five frequently detected pathogens were MP (8.6\%), PIV (8.4\%), RSV (7.5\%), flu (6.6\%), HRV (6.1\%). For the 274 SCAP children, the most five frequently detected pathogens were PIV (10.2\%), RSV (8.4\%), HRV (8\%), MP (6.6\%), and flu (4.0\%) (Figure 3).

Of 4,083 CAP adults, the most five frequently detected pathogens were flu (9.0\%), MP (5.0\%), HRV (3.1\%), PIV (2.8\%), CoV (2.2\%). For the 797 adults with SCAP, the most five commonly detected pathogens were flu (8.9\%), PIV (3.8\%), HRV (3.1\%), RSV (2.4\%), and CoV $(2.3 \%)$. The detection rate of MP in SCAP patients was significantly lower than that in NSCAP patients $(6.1 \% v s$. $0.8 \%, \mathrm{P}<0.001$ ) (Figure 3).

Detection of viral pathogens among CAP patients decreased steadily with age (Figure 4). Flu was more frequently detected among persons $\geq 10$ years old than in younger children ( $8.9 \%$ vs. $6.7 \%, \mathrm{P}=0.005)$. RSV was more commonly detected among children $<5$ years old than older persons $(10.0 \%$ vs. $2.0 \%, \mathrm{P}<0.001)$. MP was more commonly detected among patients between the ages of 5-44 years old than other age groups $(17.2 \%$ vs. $2.4 \%, \mathrm{P}<0.001)$. PIV and HRV were more frequently detected among children than among adults (PIV: $8.4 \%$ vs. $2.8 \%, \mathrm{P}<0.001$; HRV: $6.1 \%$ vs. $3.1 \%, \mathrm{P}<0.001)$. For the SCAP patients, the similar findings were observed, but, as compared to the NSCAP, the detection of MP in adults aged 18-44 years old decreased significantly (17.4\% vs. $3.2 \%, \mathrm{P}=0.038$ ) (Figure 4, Table S2).

\section{Seasonality}

The overall detection rate of the viral pathogens among CAP patients was increased during winter and spring, and hit a minor peak between August and September. The detection rate of RSV peaked in winter, and those of FLU and HMPV were increased during winter and spring. In contrast, the detection rate of PIV rose from the spring, was high through the summer and fell in autumn. The detection rate of MP rose from the summer, remained high through the fall and fell in winter. There is no strong seasonal pattern in HRV; it was detected during the whole year (Figure 5).

\section{Risk factors of SCAP and death in hospital}

Of 6,008 CAP patients, only 5,030 (84\%) have completed their questionnaires as required, and were assessed for risk factors of SCAP and death in hospital. The univariate analyses showed that the independent predictive factors for SCAP were female, an age of 45 years or older, an age of 17 years or younger, chronic lung disease, cardiovascular disease, diabetes mellitus, chronic kidney disease, neurologic disorder, immunosuppression, MP and other system diseases. None of the nine viruses (Flu, RSV, HRV, AdV, $\mathrm{HBoV}, \mathrm{EV}, \mathrm{HMPV}, \mathrm{PIV}$, and $\mathrm{CoV}$ ) or CP were included into the predictors (Table S3). However, multivariate analysis showed that the most important independent risk factors were an age of 45 years or older (especially an age of 65 years or older), an age of 17 years or younger (especially an age of 9 years or younger), neurologic disorder, chronic renal disease and immunosuppression. Female gender was excluded from the multivariate logistic regression model 
Table 1 Demographic and clinical characteristics of 6,008 patients with community-acquired pneumonia in the study

\begin{tabular}{|c|c|}
\hline Characteristic & Value \\
\hline \multicolumn{2}{|l|}{ Age } \\
\hline Median [interquartile range], years & $52[7-75]$ \\
\hline \multicolumn{2}{|l|}{ Sub group (years), n (\%) } \\
\hline $0-1$ & $449(7.5)$ \\
\hline $2-4$ & $748(12.5)$ \\
\hline $5-9$ & $510(8.5)$ \\
\hline $10-17$ & $218(3.6)$ \\
\hline $18-44$ & $805(13.4)$ \\
\hline $45-64$ & $1,085(18.1)$ \\
\hline $65-79$ & $1,190(19.8)$ \\
\hline$\geq 80$ & $1,003(16.7)$ \\
\hline Female sex, n (\%) & $2,422(40.3)$ \\
\hline \multicolumn{2}{|l|}{ Smoke, n (\%) } \\
\hline Current smoker ${ }^{1}$ & $549(9.1)$ \\
\hline Non smoker & $4,601(76.6)$ \\
\hline Undocumented & $858(14.3)$ \\
\hline \multicolumn{2}{|l|}{ Underlying disease ${ }^{2}, \mathrm{n}(\%)$} \\
\hline Any & $2,410(40.1)$ \\
\hline Chronic lung disease & $987(16.4)$ \\
\hline Cardiovascular disease & $1,081(18.0)$ \\
\hline Chronic renal disease & $679(11.3)$ \\
\hline Neurologic disorder & $417(6.9)$ \\
\hline Chronic liver disease & $81(1.3)$ \\
\hline Diabetes mellitus & $679(11.3)$ \\
\hline Immunosuppression & $112(1.9)$ \\
\hline Other system disease & $485(8.1)$ \\
\hline Undocumented & $978(16.3)$ \\
\hline \multicolumn{2}{|l|}{ Maximum body temperature $\left({ }^{\circ} \mathrm{C}\right), \mathrm{n}(\%)$} \\
\hline$<36$ & $27(0.4)$ \\
\hline $36-37.2$ & $932(15.5)$ \\
\hline $37.3-38$ & $1,071(17.8)$ \\
\hline $38.1-39$ & $2,419(40.3)$ \\
\hline$\geq 39.1$ & $1,424(23.7)$ \\
\hline Undocumented & $135(2.2)$ \\
\hline \multicolumn{2}{|l|}{ Symptom, n (\%) } \\
\hline Cough & $5,425(90.3)$ \\
\hline Chest pain & $422(7.0)$ \\
\hline Dyspnea or tachypnea & $1,629(27.1)$ \\
\hline Moist rales & $2,549(42.4)$ \\
\hline
\end{tabular}

Table 1 (continued)
Table 1 (continued)

\begin{tabular}{lc}
\hline Characteristic & Value \\
\hline Radiographic findings, $\mathrm{n}(\%)$ & $2,107(35.1)$ \\
Consolidation or infiltrate & $447(7.4)$ \\
Pleural effusion & $3,454(57.5)$ \\
Other & \\
Severity of pneumonia, $\mathrm{n}(\%)$ & \\
NSCAP & $4,937(82.2)$ \\
SCAP & $1,071(17.8)$ \\
Hospital treatment, $\mathrm{n}(\%)$ & \\
Intensive care unit admission & $695(11.6)$ \\
Invasive mechanical ventilation & $617(10.3)$ \\
Extracorporeal membrane oxygenation & $71(1.2)$ \\
Oxygen therapy & $2,331(38.8)$ \\
Death in hospital & $168(2.8)$ \\
Patients with eligible SFLRT & \\
Age median [interquartile range], years & $1,182(19.7)$ \\
Underlying disease, $\mathrm{n}$ (\%) & $59[29-76]$ \\
SCAP, $\mathrm{n}$ (\%) & $588(49.7)$ \\
Patients without eligible SFLRT & $252(21.3)$ \\
Age Median [interquartile range], years & $4,826(80.3)$ \\
Underlying disease, $\mathrm{n}(\%)$ & $49-822(37.8)$ \\
SCAP, $\mathrm{n}$ (\%) & $819(17.0)$ \\
\hline
\end{tabular}

${ }^{1}$, smoker was defined as a person who smoked at least once a day, and this condition lasted for more than 6 months.

${ }^{2}$, any underlying medical condition included chronic lung diseases (asthma, reactive airway diseases, chronic obstructive pulmonary disease, or obstructive sleep apnea), chronic heart disease (i.e., congenital heart disease for children, coronary artery disease or congestive heart failure, but not hypertension), immunosuppression (either due to chronic condition or medication, cancer, or HIV infection with a CD4 cell count of $>200$ per cubic millimeter), diabetes mellitus, chronic kidney disease (with or without dialysis), chronic liver disease (hepatitis, cirrhosis, or hepatic failure), neurologic disorder (epilepsy, cerebral palsy, dementia, Parkinson's disease, or history of stroke), and other system disease that affected less than $1 \%$ of patients. Categories were not mutually exclusive and therefore do not sum to $100 \%$. $^{3}$, denote those cases who had radiographic diagnosis of CAP, but no detailed radiographic findings provided in their questionnaires. ${ }^{4}$, SFLRT indicates specimen obtained from lower respiratory tract, including eligible sputum, bronchoalveolar-lavage, endotracheal aspirate. There are significant differences in age media $(P<0.001)$, underlying disease rate $(P<0.001)$ and SCAP percentage $(P<0.001)$ between the group with eligible SFLRT and the group without eligible SFLRT. Notably, those cases with the missing values of underlying diseases were not included into calculation for underlying disease rates. 


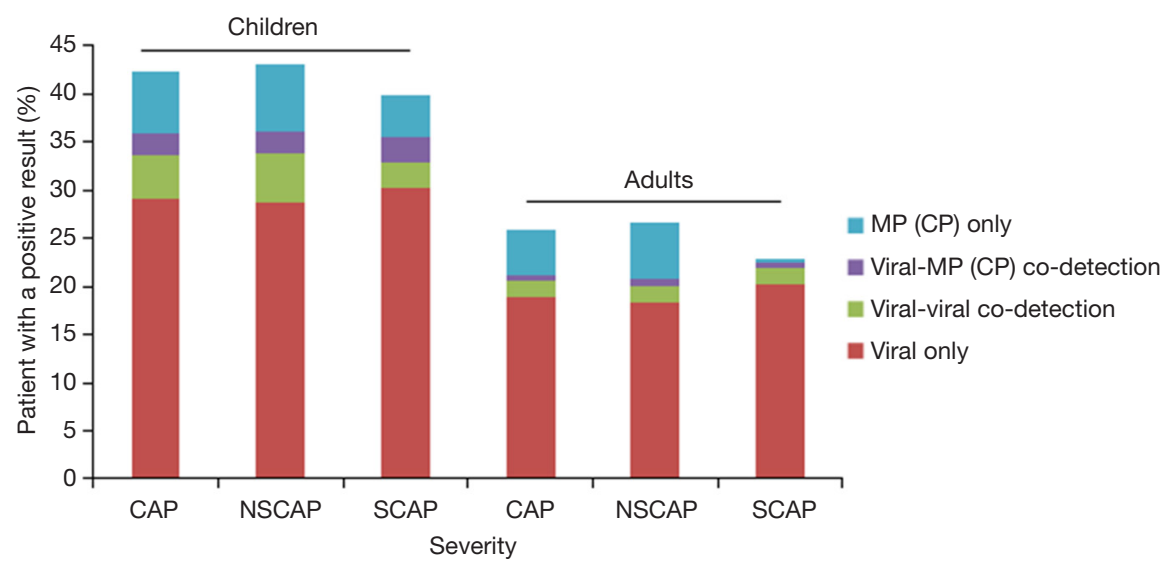

Figure 2 Distribution of infection types among CAP patients by age group and illness severity. This figure shows the results among 6,008 CAP consisting of 4,937 patients with NSCAP, and 1,071 SCAP obtained from August 1, 2014, through July 31, 2016. CAP, communityacquired pneumonia; NSCAP, non-severe CAP; SCAP, severe CAP.

A

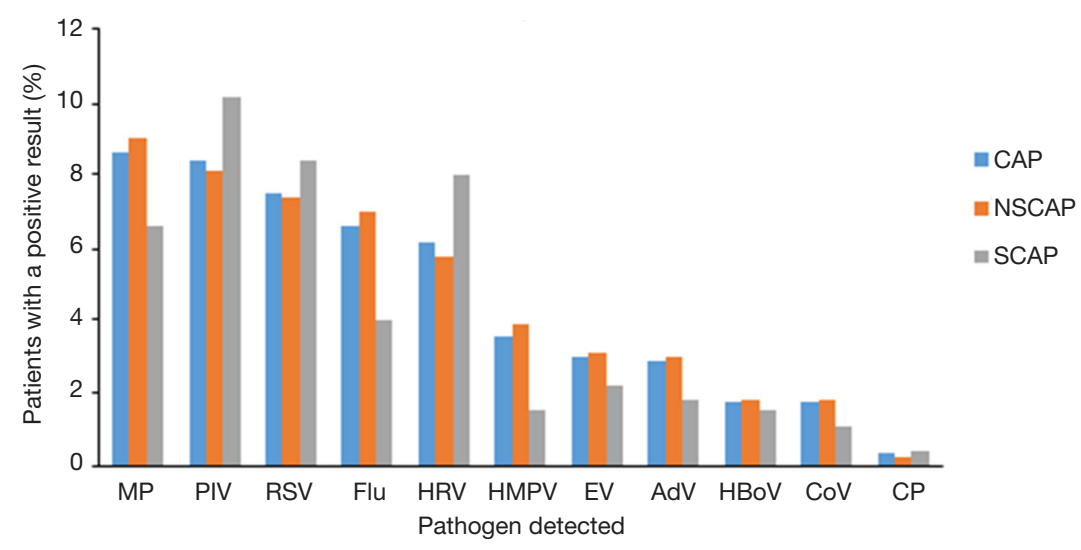

B

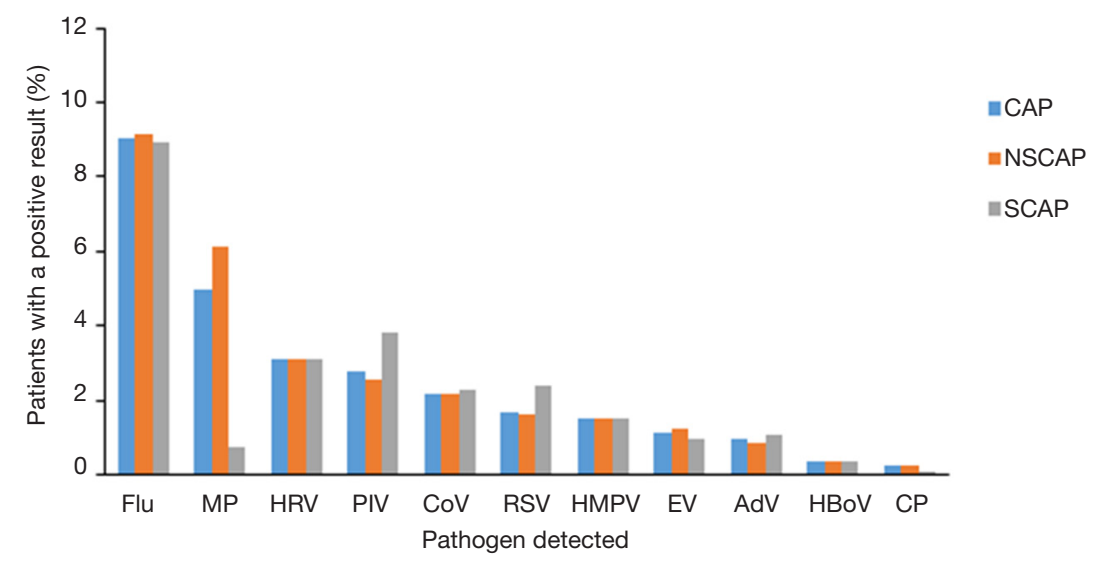

Figure 3 Percentages of the pathogens detected among (A) child patients and (B) adult patients. Among 6,008 CAP patients who had tests for Mycoplasma pneumoniae, Chlamydophila pneumoniae and viral pathogens. CAP, community-acquired pneumonia; AdV, adenovirus; $\mathrm{CoV}$, coronavirus; Flu, influenza A or B virus; HMPV, human metapneumovirus; HRV, human rhinovirus; PIV, parainfluenza virus; RSV, respiratory syncytial virus; $\mathrm{HBoV}$, human bocavirus; EV, enterovirus; MP, Mycoplasma pneumoniae; CP, Chlamydophila pneumoniae. 
A

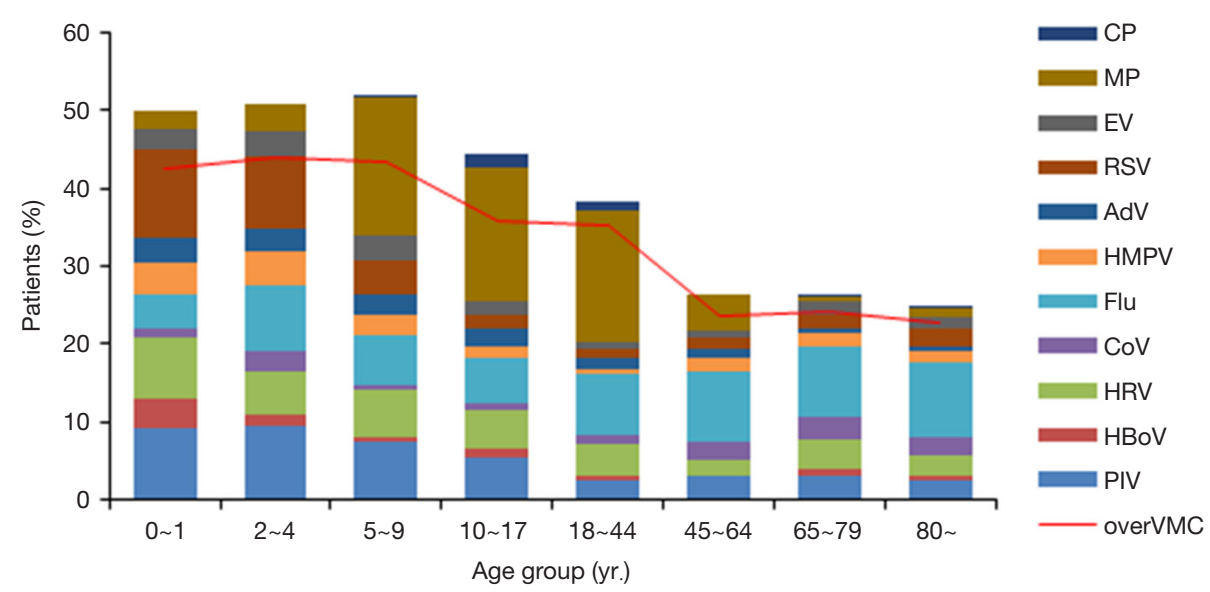

B

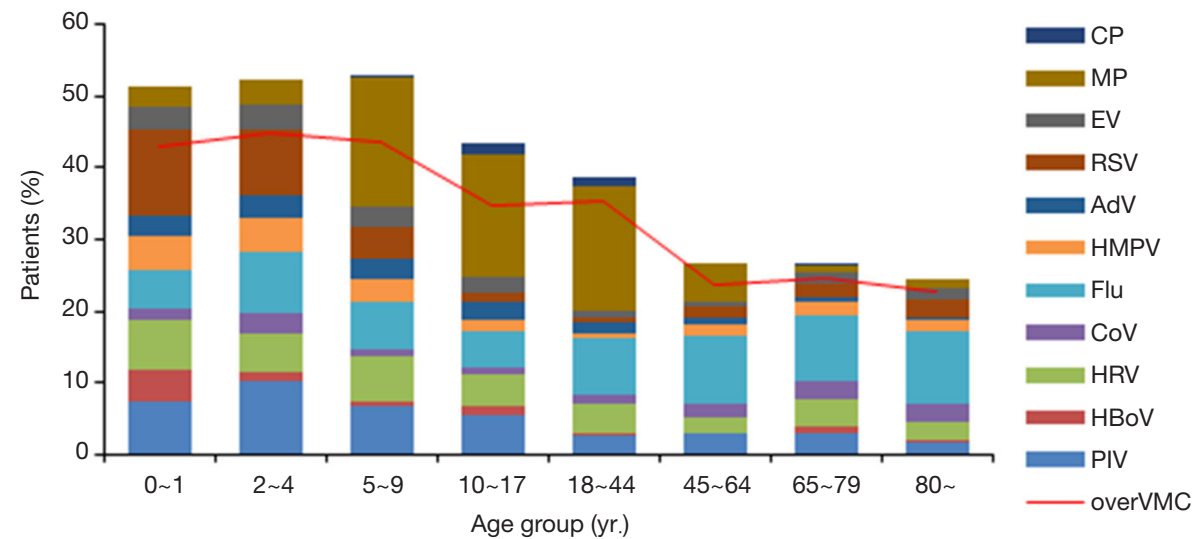

C

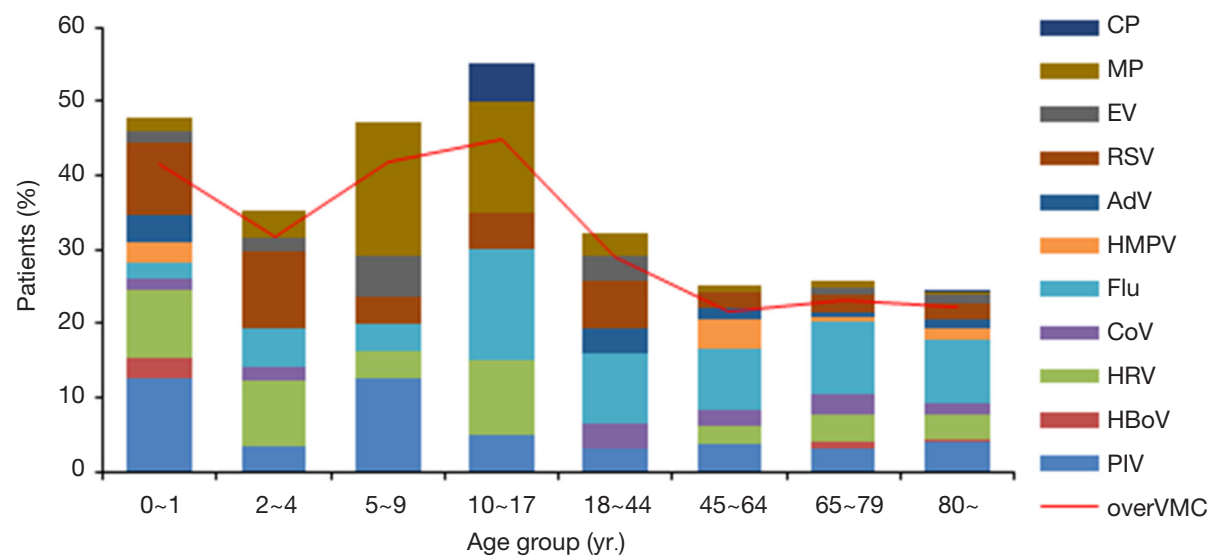

Figure 4 Spectrum of pathogens by age group among (A) all CAP patients, (B) NSCAP patients and (C) SCAP patients. Bars indicate the detection yield of each pathogen; line indicates the detection yield of pathogens together. Over VMC stands for the overall positive rate of respiratory viruses, MP and CP, It means the rate of the patients with at least one of 9 respiratory viruses, MP and CP detected. CAP, community-acquired pneumonia; NSCAP, non-severe CAP; SCAP, severe CAP. 
A

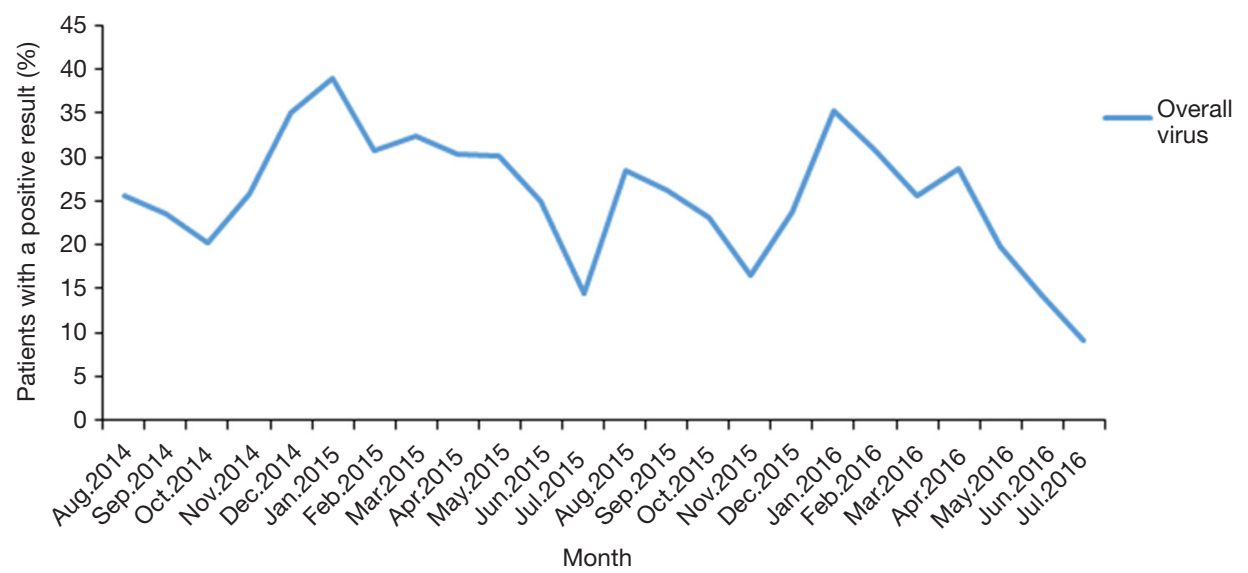

B

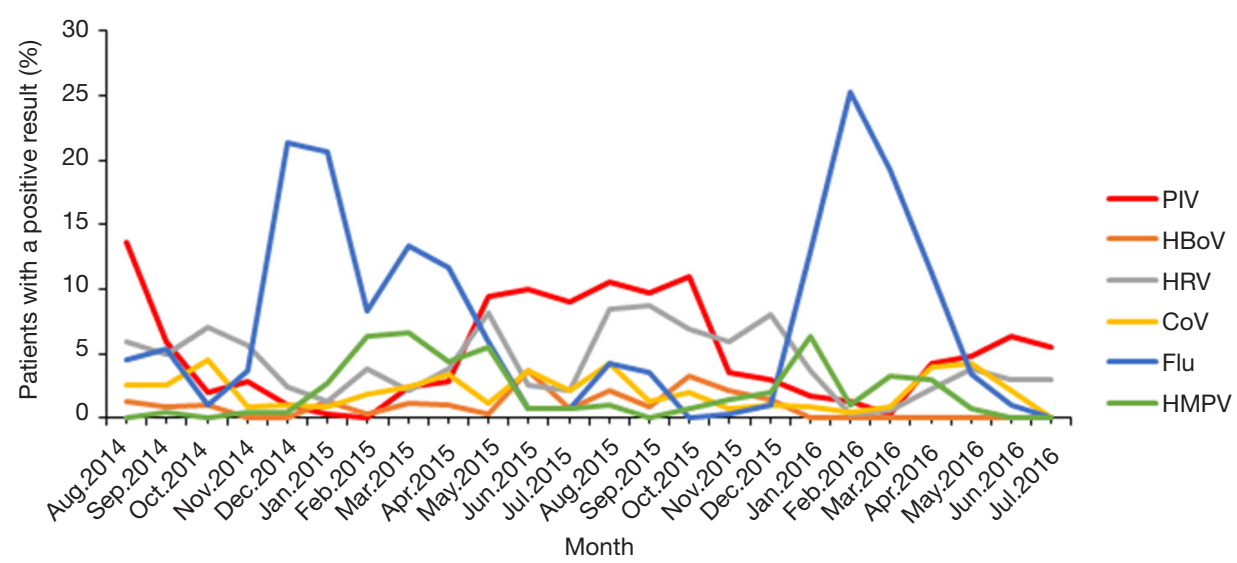

C

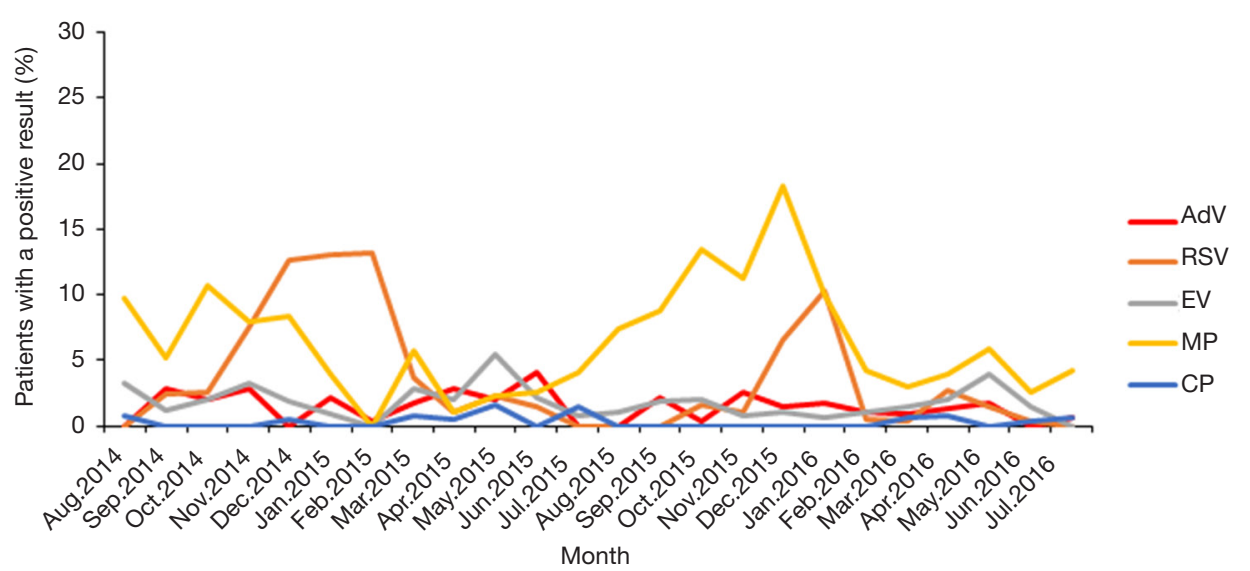

Figure 5 Monthly detection of pathogens among the CAP patients detected from August 1, 2014 to July 31, 2016. (A) Monthly detection rate of 9 viruses together among all CAP patients; (B) the frequency of patients in whom parainfluenza virus, human bocavirus, human rhinovirus, coronavirus, influenza virus, and human metapneumovirus were detected; (C) the frequency of patients in whom adenovirus, respiratory syncytial virus, enterovirus, $M$. pneumoniae, and $C$. pneumoniae were detected. Note the difference in scale of the y axis for each line graph. CAP, community-acquired pneumonia. 
Table 2 Multivariate analysis of risk factors for SCAP and death in Hospital of 5,030 CAP patients

\begin{tabular}{|c|c|c|}
\hline Risk factors & Odds ratio $(95 \% \mathrm{Cl})$ & $P$ value \\
\hline \multicolumn{3}{|l|}{ SCAP as outcome } \\
\hline \multicolumn{3}{|l|}{ Age group* (years) } \\
\hline $0-1$ & $10.80(7.06-16.54)$ & $<0.001$ \\
\hline $2-4$ & $1.90(1.20-3.01)$ & 0.006 \\
\hline $5-9$ & $3.03(1.91-4.83)$ & $<0.001$ \\
\hline $10-17$ & $2.29(1.27-4.14)$ & 0.006 \\
\hline $45-64$ & $2.06(1.36-3.14)$ & 0.001 \\
\hline $65-79$ & $3.50(2.33-5.25)$ & $<0.001$ \\
\hline$\geq 80$ & $4.39(2.91-6.63)$ & $<0.001$ \\
\hline Chronic lung disease & $1.74(1.45-2.09)$ & $<0.001$ \\
\hline Cardiovascular disease & $1.55(1.29-1.85)$ & $<0.001$ \\
\hline Diabetes mellitus & $1.55(1.27-1.90)$ & $<0.001$ \\
\hline Chronic kidney disease & $2.52(1.80-3.53)$ & $<0.001$ \\
\hline Immunosuppression & $2.41(1.59-3.67)$ & $<0.001$ \\
\hline Neurologic disorder & $3.19(2.54-4.01)$ & $<0.001$ \\
\hline Other system disease & $1.29(1.02-1.63)$ & 0.033 \\
\hline Mycoplasma pneumoniae & $0.61(0.38-0.96)$ & 0.031 \\
\hline Constant & 0.05 & $<0.001$ \\
\hline
\end{tabular}

Death in hospital as outcome

Age group* (years)

$\begin{array}{lcc}0-1 & 0(0-0) & 0.994 \\ 2-4 & 0(0-0) & 0.992 \\ 5-9 & 0(0-0) & 0.993 \\ 10-17 & 0.78(0.09-7.08) & 0.828 \\ 45-64 & 2.68(0.90-8.01) & 0.078 \\ 65-79 & 7.85(2.83-21.83) & <0.001 \\ \geq 80 & 10.09(3.62-28.06) & <0.001 \\ \text { Diabetes mellitus } & 1.44(1.01-2.06) & 0.005 \\ \text { Chronic kidney disease } & 2.06(1.25-3.41) & <0.001 \\ \text { Immunosuppression } & 6.52(4.00-10.62) & 0.023 \\ \text { Neurologic disorder } & 1.57(1.06-2.33) & <0.001 \\ \text { Constant } & 0 & 0.994\end{array}$

*, age group was transferred to dummy variables with 18-45 years as the reference category. CAP, community-acquired pneumonia; SCAP, severe CAP.
(Table 2).

The univariate analyses showed that the risk factors of death in Hospital were an age of 45 years or older and an age of 17 years or younger; chronic lung disease, cardiovascular disease, diabetes mellitus, chronic kidney disease, neurological disorder, immunosuppression and MP. Almost all aforementioned pathogens except MP were excluded (Table S4). However, multivariate analysis showed that the independent risk factors only included an age of 65 years or older, chronic kidney disease, neurologic disorder immunosuppression (Table 2).

\section{Discussion}

This study was one of the largest multiple center surveillance projects on the etiology of CAP among the Chinese to date, and mainly investigated infections of viruses, $\mathrm{MP}$ and $\mathrm{CP}$ associated with CAP.

In this study, the most five commonly detected viruses were PIV (8.4\%), RSV (7.5\%), flu (6.6\%), HRV (6.1\%) and HMPV (3.6\%) among children, and flu $(9.0 \%)$, HRV (3.1\%), PIV (2.8\%), CoV (2.2\%) and RSV $(1.7 \%)$ among adults. However, Jain et al. $(5,6)$ reported the top five frequently detected viruses were RSV (28.0\%), HRV (27.3\%), HMPV (12.8\%), AdV (11.2\%) and PIV (6.8\%) among children with CAP requiring hospitalization; and HRV (8.4\%), flu (5.7\%), HMPV (3.8\%), RSV (2.9\%), PIV (2.9\%) among adults with CAP requiring hospitalization in USA between 2010-2013. Xie et al. (9) reported that the most three commonly detected viruses were RSV (4.5-50.9\%), HRV (12.9-36.2\%), PIV (3.9-17.8\%) among children younger than 15 years old with acute lower respiratory tract infection from 2007 to 2010. The detection rates of pathogens of this study were obviously lower than those of Jain's (except for PIV among CAP children), but consistent to Xie's findings (except for HRV among CAP children). The difference in inclusion criteria of study populations may be one of the important reasons for the different detection rates. Both CAP inpatient and CAP outpatient were enrolled in our study, but only CAP inpatients were included in Jain's study. Furthermore geographic difference between America and China should also be considered. Our findings indicated that PIV and HRV, which were usually considered as the common pathogens of the patients with acute upper respiratory tract infection, were also the important pathogens of the patients with CAP in China, and should be paid more attention to.

Our results showed that M. Pneumoniae was the most 
frequently detected pathogen in CAP children (8.6\%) and the second in CAP adults (5.0\%). Liu et al. (7) reported that $M$. pneumoniae was detected among $13.4 \%$ of CAP adults in China in 2004. Tao et al. (8) reported that $M$. pneumoniae was detected among $31.1 \%$ of CAP adults in China from 2004 to 2005. Although the detection rates of M. pneumoniae varied considerably among these studies, likely due to a difference in testing methods and study populations, all of the studies indicated that $M$. pneumoniae was one of the very common pathogens of CAP patients in China, as was one of the important characteristics of CAP in China (10). Notably, M. Pneumoniae was more commonly detected among patients between the ages of 5-44 years old than other age groups, and the detection rate of $M$. Pneumoniae in adult SCAP was significantly lower than that in adult NSCAP, which indicate that M. Pneumoniae infection seems more likely to occur in more "healthy" population. The reason behind this finding is unclear and needs the further research.

The multivariate analyses show that the most important risk factors for SCAP were an age of 45 years or older (especially an age of 65 years or older), an age of 17 years or younger (especially an age of 9 years or younger), chronic renal disease, neurological disorder and immunosuppression. None of respiratory viruses, MP and CP was included. The independent predictors of death in hospital were an age of 65 years or older, chronic kidney disease, neurologic disorders and immunosuppression. However, an age of 9 years or younger, which was one of the important risk factors of SCAP, was not included. Zhang et al. (14) also found that fatality of child SCAP among Chinese was associated with congenital heart disease, trisomy 21 syndrome, cerebral palsy, and immune deficiency, and no relationship was noted between fatality and specific pathogens. Their findings were consistent to ours. Wang et al. (15) reported that HRV was associated with severe adult CAP in Chinese; Yu et al. (16) reported that human adenovirus 7 type caused higher fatality rate $(28.6 \%)$ of Chinese child acute respiratory infectious disease than other types. However, their conclusions were almost drew from univariate analyses, and some confounding factors, such as underlying disease, should be excluded.

Despite our maximum effort, there were still some limitations in this study.

First, bacterial pathogens were not tested, though they are very important to the etiology of CAP, especially in adult patients. This lack of bacterial testing results, not only led to an incomplete study on the etiology of CAP, but also made it impossible to determine whether or not there is an association between bacterial pathogens and SCAP.

Second, as some studies reported (5), many respiratory viruses identified in patients with CAP might just be colonisation other than the causative pathogens. However, respiratory virus colonisation is not so common as respiratory bacteria. According to the Guideline on the Management of Community Acquired Pneumonia among Children in China (released in 2013 by Chinese pediatric society, Chinese Medicine Association), virus identified from respiratory specimen could be thought as the causative pathogen of the respiratory infection.

Finally, the study population in this study was from Beijing, one of the most developed areas of China, which made it difficult to apply all conclusions of this study to China, as a whole, given the big difference in characteristics of people, culture, economy, geography and climate among the different areas of China.

\section{Conclusions}

In conclusion, the above mentioned 11 atypical pathogens were the common causes of CAP (including SCAP) among Chinese, however, among those patients who were exposed to these 11 atypical respiratory pathogens (influenza virus $\mathrm{H} 5 \mathrm{~N} 1$, influenza virus H7N9 and coronavirus SARS not included), the increase of the risk for SCAP was not observed. The important risk factors for SCAP were an age of 9 years or younger, an age of 65 years or older and coexisting disease (immunosuppression, neurologic disorders and cardiovascular disease). These factors, except for having an age of 9 years or younger, were significantly associated with death in hospital. These findings suggest that the patient's health condition may play a more important role during the SCAP development process than these 11 atypical pathogens.

\section{Acknowledgements}

We would like to thank the physicians from 30 sentinel hospitals of RPSS in Beijing for collecting sample and investigating cases. We also thank the epidemiological investigators and laboratory personnel from 16 district level CDCs for delivering samples and testing assistance, and thank Dr. Jim Zhang and Dr. Hongxing Zhao of Public Health England for their advice on manuscript preparation. Funding: This work was supported by the Beijing Municipal Science and Technology Commission (Z151100003915140), 
and National Major Science and Technology Project for Control and Prevention of Major Infectious Diseases of China (2016ZX10004206).

\section{Footnote}

Conflicts of Interest: The authors have no conflicts of interest to declare.

Ethical Statement: The ethics approvals for the protocol of this study were obtained from the Ethics Committee of Beijing Center for Disease Prevention and Control. Before enrollment, the nature, purpose, procedures, potential healthy impact and benefits of this study were explained carefully to each patient or their care providers, and written informed consents were obtained. Patients themselves were required to provide assents when their age and medical condition were appropriate.

\section{References}

1. Schnoor M, Hedicke J, Dalhoff K, et al. Approaches to estimate the population-based incidence of community acquired pneumonia. J Infect 2007;55:233-9.

2. Marston BJ, Plouffe JF, File TM Jr, et al. Incidence of community-acquired pneumonia requiring Hospitalization. Results of a population-based active surveillance Study in Ohio. The Community-Based Pneumonia Incidence Study Group. Arch Intern Med 1997;157:1709-18.

3. Fielden NM. Community-acquired pneumonia. Perspect Respir Nurs 1998;9:1-2, 4, 6 passim.

4. Takaki M, Nakama T, Ishida $M$, et al. High incidence of community-acquired pneumonia among rapidly aging population in Japan: a prospective Hospital-based surveillance. Jpn J Infect Dis 2014;67:269-75.

5. Jain S, Self WH, Wunderink RG, et al. CommunityAcquired Pneumonia Requiring Hospitalization among U.S. Adults. N Engl J Med 2015;373:415-27.

6. Jain S, Williams DJ, Arnold SR, et al. Communityacquired pneumonia requiring Hospitalization among U.S. children. N Engl J Med 2015;372:835-45.

Cite this article as: Gong C, Zhang T, Luo M, Li A, Dong M, Li M, Wang Y, Huang F. Distribution of the atypical pathogens of community-acquired pneumonia to disease severity. J Thorac Dis 2018;10(11):5991-6001. doi: 10.21037/jtd.2018.10.50
7. Liu Y, Chen M, Zhao T, et al. Causative agent distribution and antibiotic therapy assessment among adult patients with community acquired pneumonia in Chinese urban population. BMC Infect Dis 2009;9:31.

8. Tao LL, Hu BJ, He LX, et al. Etiology and antimicrobial resistance of community-acquired pneumonia in adult patients in China. Chin Med J (Engl) 2012;125:2967-72.

9. Xie ZD, Xiao Y, Liu CY, et al. Three years surveillance of viral etiology of acute lower respiratory tract infection in children from 2007 to 2010. Zhonghua Er Ke Za Zhi 2011;49:745-9.

10. Chinese Thoracic Society CMA. Guidelines on diagnosis and treatment for community acquired pneumonia among adult in China. Chinese Practical Journal of Rural Doctor 2013;20:11-5.

11. Chinese Pediatric Society, Chinese Medical Association. Guidelines on management of children with community acquired pneumonia in China (revised 2013, the first). Chinese Journal of Pediatrics 2013;51:745-52.

12. Chinese Pediatric Society, Chinese Medical Association. Guidelines on management of children with community acquired pneumonia (revised in 2013, the last). Chinese Journal of Pediatrics 2013;51:856-62.

13. Mandell LA, Wunderink RG, Anzueto A, et al. Infectious Diseases Society of America/American Thoracic Society consensus guidelines on the management of communityacquired pneumonia in adults. Clin Infect Dis 2007;44 Suppl 2:S27-72.

14. Zhang Q, Guo Z, Bai Z, et al. A 4 year prospective study to determine risk factors for severe community acquired pneumonia in children in southern China. Pediatr Pulmonol 2013;48:390-7.

15. Wang K, Xi W, Yang D, et al. Rhinovirus is associated with severe adult community-acquired pneumonia in China. J Thorac Dis 2017;9:4502-11.

16. Yu Z, Zeng Z, Zhang J, et al. Fatal Community-acquired Pneumonia in Children Caused by Re-emergent Human Adenovirus 7d Associated with Higher Severity of Illness and Fatality Rate. Sci Rep 2016;6:37216. 
Table S1 Thirty sentinel hospitals from all of 16 districts in Beijing, China

\begin{tabular}{|c|c|}
\hline Districts & Sentinel hospitals \\
\hline Dongcheng district & Tiantan Hospital, Tongren Hospital, Beijing No. 6 Hospital, Dongzhimen Hospital \\
\hline Xicheng district & Peking University People's Hospital, Beijing Children' Hospital, Jianggong Hospital \\
\hline Chaoyiang district & The First Hospital of Qsinghua University, China-Japan friendship Hospital, Chuiyangliu Hospital \\
\hline Haidian district & Aerospace Center Hospital, Beijing Shijitan Hospital, Haidian Hospital \\
\hline Shijingshan district & Shijinshan Hospital, Peking University Shougang Hospital, Jingxi Court of Chuiyangliu Hospital \\
\hline Miyun district & Miyun Hospital \\
\hline Daxin district & Daxing Hospital \\
\hline Mentougou district & Beijing Jingmei Group General Hospital \\
\hline Changping district & Changping Hospital \\
\hline Huairou district & Huairou First Hospital \\
\hline Fengtai district & Dongfang Hospital \\
\hline Fangshan district & Liangxiang Hospital \\
\hline Shunyi district & Shunyi Hospital \\
\hline Tongzhou district & Luhe Hospital \\
\hline Pinggu district & Pinggu Hospital \\
\hline Yanqing district & Yianqing Hospital \\
\hline
\end{tabular}

Table S2 Number (percentage) of patients with a positive result by illness severity and age group

\begin{tabular}{|c|c|c|c|c|c|c|c|c|c|c|c|c|c|c|}
\hline \multirow{2}{*}{ Illness severity } & \multirow{2}{*}{$\begin{array}{l}\text { Age group } \\
\text { (years) }\end{array}$} & \multirow{2}{*}{ Cases } & \multicolumn{12}{|c|}{ Patients with a positive result (\%) } \\
\hline & & & PIV & HBoV & HRV & Cov & Flu & HMPV & AdV & RSV & EV & MP & $\mathrm{CP}$ & Over \\
\hline \multirow[t]{9}{*}{ CAP $(n=6,008)$} & $0-1$ & 449 & $41(9.1)$ & $17(3.8)$ & $35(7.8)$ & $6(1.3)$ & $20(4.5)$ & $18(4.0)$ & $14(3.1)$ & 51 (11.4) & $12(2.7)$ & $11(2.4)$ & $0(0)$ & $191(42.5)$ \\
\hline & $2-4$ & 748 & $72(9.6)$ & $10(1.3)$ & $42(5.6)$ & $20(2.7)$ & $62(8.3)$ & $34(4.5)$ & $22(2.9)$ & $69(9.2)$ & $25(3.3)$ & $27(3.6)$ & $0(0)$ & $328(43.9)$ \\
\hline & $5-9$ & 510 & $37(7.3)$ & $4(0.8)$ & $30(5.9)$ & $4(0.8)$ & $32(6.3)$ & $14(2.7)$ & $14(2.7)$ & $21(4.1)$ & $17(3.3)$ & $91(17.8)$ & $1(0.2)$ & $222(43.5)$ \\
\hline & $10-17$ & 218 & $12(5.5)$ & $2(0.9)$ & $11(5.0)$ & $2(0.9)$ & $13(6.0)$ & $3(1.4)$ & $5(2.3)$ & $4(1.8)$ & $4(1.8)$ & $37(17)$ & $4(1.8)$ & $78(35.8)$ \\
\hline & $18-44$ & 805 & $21(2.6)$ & $4(0.5)$ & $32(4.0)$ & $10(1.2)$ & $63(7.8)$ & $5(0.6)$ & $13(1.6)$ & $8(1.0)$ & $8(1.0)$ & $136(16.9)$ & $10(1.2)$ & 283 (35.2) \\
\hline & $45-64$ & 1,085 & $32(2.9)$ & $1(0.1)$ & $23(2.1)$ & $24(2.2)$ & $100(9.2)$ & $20(1.8)$ & $12(1.1)$ & $16(1.5)$ & $8(0.7)$ & $51(4.7)$ & $0(0)$ & $256(23.6)$ \\
\hline & $65-79$ & 1,190 & $37(3.1)$ & $9(0.8)$ & $45(3.8)$ & $33(2.8)$ & 110 (9.2) & $19(1.6)$ & $7(0.6)$ & $23(1.9)$ & $19(1.6)$ & $10(0.8)$ & $1(0.1)$ & $287(24.1)$ \\
\hline & $\geq 8 \mathrm{yr}$ & 1,003 & $26(2.6)$ & $3(0.3)$ & $28(2.8)$ & $22(2.2)$ & $96(9.6)$ & $16(1.6)$ & $7(0.7)$ & $23(2.3)$ & $15(1.5)$ & $9(0.9)$ & $1(0.1)$ & 227 (22.6) \\
\hline & Total & 6,008 & $278(4.6)$ & $50(0.8)$ & $246(4.1)$ & $121(2.0)$ & $496(8.3)$ & $129(2.1)$ & $94(1.6)$ & 215 (3.6) & 108 (1.8) & $372(6.2)$ & $17(0.3)$ & $1,872(31.2)$ \\
\hline \multirow[t]{9}{*}{$\operatorname{NSCAP}(n=4,937)$} & $0-1$ & 307 & $23(7.5)$ & $13(4.2)$ & $22(7.2)$ & $4(1.3)$ & $17(5.5)$ & $14(4.6)$ & $9(2.9)$ & $37(12.1)$ & $10(3.3)$ & $8(2.6)$ & $0(0)$ & $132(43)$ \\
\hline & $2-4$ & 691 & $70(10.1)$ & $10(1.4)$ & $37(5.4)$ & $19(2.7)$ & $59(8.5)$ & $34(4.9)$ & $22(3.2)$ & $63(9.1)$ & $24(3.5)$ & $25(3.6)$ & $0(0)$ & 310 (44.9) \\
\hline & $5-9$ & 455 & $30(6.6)$ & $4(0.9)$ & $28(6.2)$ & $4(0.9)$ & $30(6.6)$ & $14(3.1)$ & $14(3.1)$ & $19(4.2)$ & $14(3.1)$ & $81(17.8)$ & $1(0.2)$ & 199 (43.7) \\
\hline & $10-17$ & 198 & $11(5.6)$ & $2(1.0)$ & $9(4.5)$ & $2(1.0)$ & $10(5.1)$ & $3(1.5)$ & $5(2.5)$ & $3(1.5)$ & $4(2.0)$ & $34(17.2)$ & $3(1.5)$ & $69(34.8)$ \\
\hline & $18-44$ & 774 & $20(2.6)$ & $4(0.5)$ & $32(4.1)$ & $9(1.2)$ & $60(7.8)$ & $5(0.6)$ & $12(1.6)$ & $6(0.8)$ & $7(0.9)$ & 135 (17.4) & $10(1.3)$ & $274(35.4)$ \\
\hline & $45-64$ & 952 & $27(2.8)$ & $1(0.1)$ & $20(2.1)$ & $21(2.2)$ & $89(9.3)$ & $15(1.6)$ & $10(1.1)$ & $13(1.4)$ & $8(0.8)$ & $50(5.3)$ & $0(0)$ & 227 (23.8) \\
\hline & $65-79$ & 887 & $27(3.0)$ & $7(0.8)$ & $34(3.8)$ & $24(2.7)$ & $81(9.1)$ & $17(1.9)$ & $5(0.6)$ & $16(1.8)$ & $16(1.8)$ & $7(0.8)$ & $1(0.1)$ & 217 (24.5) \\
\hline & $\geq 80$ & 673 & $12(1.8)$ & $2(0.3)$ & $17(2.5)$ & $17(2.5)$ & $68(10.1)$ & $11(1.6)$ & $3(0.4)$ & $16(2.4)$ & $11(1.6)$ & $8(1.2)$ & $0(0)$ & $153(22.7)$ \\
\hline & Total & 4,937 & $220(4.5)$ & $43(0.9)$ & $199(4.0)$ & $100(2.0)$ & $414(8.4)$ & $113(2.3)$ & $80(1.6)$ & $173(3.5)$ & $94(1.9)$ & $348(7.0)$ & $15(0.3)$ & $1,581(32)$ \\
\hline \multirow[t]{9}{*}{$\operatorname{SCAP}(n=1,071)$} & $0-1$ & 142 & $18(12.7)$ & $4(2.8)$ & $13(9.2)$ & $2(1.4)$ & $3(2.1)$ & $4(2.8)$ & $5(3.5)$ & $14(9.9)$ & $2(1.4)$ & $3(2.1)$ & $0(0)$ & $59(41.5)$ \\
\hline & $2-4$ & 57 & $2(3.5)$ & $0(0)$ & $5(8.8)$ & $1(1.8)$ & $3(5.3)$ & $0(0)$ & $0(0)$ & $6(10.5)$ & $1(1.8)$ & $2(3.5)$ & $0(0)$ & $18(31.6)$ \\
\hline & $5-9$ & 55 & $7(12.7)$ & $0(0)$ & $2(3.6)$ & $0(0)$ & $2(3.6)$ & $0(0)$ & $0(0)$ & $2(3.6)$ & $3(5.5)$ & $10(18.2)$ & $0(0)$ & $23(41.8)$ \\
\hline & $10-17$ & 20 & $1(5.0)$ & $0(0)$ & $2(10.0)$ & $0(0)$ & $3(15.0)$ & $0(0)$ & $0(0)$ & $1(5.0)$ & $0(0)$ & $3(15.0)$ & $1(5.0)$ & $9(45.0)$ \\
\hline & $18-44$ & 31 & 1 (3.2) & $0(0)$ & $0(0)$ & 1 (3.2) & $3(9.7)$ & $0(0)$ & 1 (3.2) & $2(6.5)$ & $1(3.2)$ & 1 (3.2) & $0(0)$ & $9(29.0)$ \\
\hline & $45-64$ & 133 & $5(3.8)$ & $0(0)$ & $3(2.3)$ & $3(2.3)$ & $11(8.3)$ & $5(3.8)$ & $2(1.5)$ & $3(2.3)$ & $0(0)$ & $1(0.8)$ & $0(0)$ & $29(21.8)$ \\
\hline & $65-79$ & 303 & $10(3.3)$ & $2(0.7)$ & $11(3.6)$ & $9(3.0)$ & $29(9.6)$ & $2(0.7)$ & $2(0.7)$ & 7 (2.3) & $3(1.0)$ & $3(1.0)$ & $0(0)$ & $70(23.1)$ \\
\hline & $\geq 80$ & 330 & $14(4.2)$ & $1(0.3)$ & $11(3.3)$ & $5(1.5)$ & $28(8.5)$ & $5(1.5)$ & $4(1.2)$ & $7(2.1)$ & $4(1.2)$ & $1(0.3)$ & $1(0.3)$ & $74(22.4)$ \\
\hline & Total & 1,071 & $58(5.4)$ & $7(0.7)$ & $47(4.4)$ & $21(2.0)$ & $82(7.7)$ & $16(1.5)$ & 14 (1.3) & $42(3.9)$ & $14(1.3)$ & $24(2.2)$ & $2(0.2)$ & 291 (27.2) \\
\hline
\end{tabular}

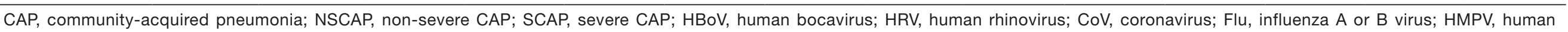
metapneumovirus; AdV, adenovirus; RSV, respiratory syncytial virus; PIV, parainfluenza virus; EV, enterovirus; MP, Mycoplasma pneumoniae; CP, Chlamydophila pneumoniae. 
Table S3 Univariate analysis of risk factors for SCAP of 5,030 CAP patients

\begin{tabular}{|c|c|c|c|}
\hline Variable & NSCAP $[3,974], \mathrm{n}(\%)$ & SCAP $[1,056], n(\%)$ & $P$ value \\
\hline Age (years) & & & $<0.001$ \\
\hline $0-1$ & $230(5.8)$ & $136(12.9)$ & \\
\hline $2-4$ & $583(14.7)$ & $56(5.3)$ & \\
\hline $10-17$ & $176(4.4)$ & $20(1.9)$ & \\
\hline $18-44$ & $560(14.1)$ & $31(2.9)$ & \\
\hline $45-64$ & $731(18.4)$ & $130(12.3)$ & \\
\hline $65-79$ & $759(19.1)$ & $301(28.5)$ & \\
\hline Chronic lung disease & $654(16.5)$ & $333(31.5)$ & $<0.001$ \\
\hline Cardiovascular disease & $686(17.3)$ & $395(37.4)$ & $<0.001$ \\
\hline Diabetes mellitus & $437(11.0)$ & 242 (22.9) & $<0.001$ \\
\hline Chronic kidney disease & $82(2.1)$ & $92(8.7)$ & $<0.001$ \\
\hline Chronic liver disease & $59(1.5)$ & $22(2.1)$ & 0.170 \\
\hline Immunosuppression & $62(1.6)$ & $50(4.7)$ & $<0.001$ \\
\hline Neurologic disorder & $210(5.3)$ & 207 (19.6) & $<0.001$ \\
\hline Other system disease & $348(8.8)$ & $137(13.0)$ & $<0.001$ \\
\hline AdV & $65(1.6)$ & $14(1.3)$ & 0.472 \\
\hline RSV & $153(3.9)$ & $41(3.9)$ & 0.961 \\
\hline PIV & $192(4.8)$ & $58(5.5)$ & 0.380 \\
\hline EV & $88(2.2)$ & $14(1.3)$ & 0.069 \\
\hline MP & $295(7.4)$ & $23(2.2)$ & $<0.001$ \\
\hline $\mathrm{CP}$ & $11(0.3)$ & $2(0.2)$ & 0.876 \\
\hline
\end{tabular}

*, only 4573 of 5,030 cases have full information on smoke history. CAP, community-acquired pneumonia; NSCAP, non-severe CAP; SCAP, severe CAP; HBoV, human bocavirus; HRV, human rhinovirus; CoV, coronavirus; Flu, influenza A or B virus; HMPV, human metapneumovirus; AdV, adenovirus; RSV, respiratory syncytial virus; PIV, parainfluenza virus; EV, enterovirus; MP, Mycoplasma pneumoniae; CP, Chlamydophila pneumoniae. 
Table S4 Univariate analysis of risk factors for death in hospital of 5,030 CAP patients

\begin{tabular}{|c|c|c|c|}
\hline Variable & Death [168], n (\%) & Recovery $[4,862], \mathrm{n}(\%)$ & $P$ value \\
\hline \multicolumn{4}{|l|}{ Age (years) } \\
\hline $0-1$ & $0(0)$ & $366(7.5)$ & $<0.001$ \\
\hline $2-4$ & $0(0)$ & $639(13.1)$ & \\
\hline $10-17$ & $1(0.6)$ & $195(4.0)$ & \\
\hline $18-44$ & $4(2.4)$ & $587(12.1)$ & \\
\hline $45-64$ & $18(10.7)$ & $843(17.3)$ & \\
\hline $65-79$ & $68(40.5)$ & $992(20.4)$ & \\
\hline Chronic lung disease & $69(41.1)$ & 918 (18.9) & $<0.001$ \\
\hline Cardiovascular disease & $79(47.0)$ & $1,002(20.6)$ & $<0.001$ \\
\hline Diabetes mellitus & $52(31.0)$ & 627 (12.9) & $<0.001$ \\
\hline Chronic kidney disease & $23(13.7)$ & $151(3.1)$ & $<0.001$ \\
\hline Chronic liver disease & $5(3.0)$ & $76(1.6)$ & 0.263 \\
\hline Immunosuppression & $27(16.1)$ & $85(1.7)$ & $<0.001$ \\
\hline Neurologic disorder & $39(23.2)$ & $378(7.8)$ & $<0.001$ \\
\hline Other system disease & $21(12.5)$ & $464(9.5)$ & 0.202 \\
\hline AdV & $0(0)$ & $79(1.6)$ & 0.177 \\
\hline RSV & $3(1.8)$ & $191(3.9)$ & 0.156 \\
\hline PIV & $3(1.8)$ & $247(5.1)$ & 0.053 \\
\hline EV & $3(1.8)$ & $99(2.0)$ & 1.000 \\
\hline MP & $0(0)$ & $318(6.5)$ & 0.001 \\
\hline $\mathrm{CP}$ & $0(0)$ & $13(0.3)$ & 1.000 \\
\hline
\end{tabular}

*, only 4,573 of 5,030 cases have full information on smoke history. CAP, community-acquired pneumonia; HBoV, human bocavirus; HRV, human rhinovirus; CoV, coronavirus; Flu, influenza A or B virus; HMPV, human metapneumovirus; AdV, adenovirus; RSV, respiratory syncytial virus; PIV, parainfluenza virus; EV, enterovirus; MP, Mycoplasma pneumoniae; CP, Chlamydophila pneumoniae. 